\title{
Norms and facts in measurement
}

\author{
J. van Brakel \\ Foundations of Physics, Physics Laboratory, State University of Utrecht, \\ PO Box 80.000, 3508 TA Utrecht, The Netherlands
}

\begin{abstract}
Publications concerned with the foundations of measurement often accept uncritically the theory/observation and the norm/fact distinction. However, measurement is measurement-in-a-context. This is analysed in the first part of the paper. Important aspects of this context are: the purpose of the particular measurement; everything we know; everything we can do; and the empirical world. Dichotomies, such as definition versus empirical law, are by themselves not very useful because the 'rules of measurement' are facts or norms depending on the context chosen or considered. In the second part of the paper, three methodological premises for a theory of measurement are advocated: reduce arbitrariness, against fundamentalism, and do not be afraid of realism.
\end{abstract}

\section{The problem of dichotomies}

In the literature on the foundations of measurement, a number of dichotomies are used in describing properties of statements about various aspects of measurement. One of the most common distinctions is that between a convention and an empirical fact, or between an (a priori) definition and an (a posteriori) empirical law. Some other dichotomies that roughly correspond to the two just mentioned are given in Table 1 . The following are examples of statements to which these dichotomies have been applied:

- Electric conductivity is the inverse of electric resistance (Krantz et al, 1971)

- Density is mass divided by volume (Krantz et al, 1971; Grunstra, 1969; Osborne, 1976)

- Force is mass times acceleration (Nagel, 1968; Palacios, 1964; Gillies, 1973)

- Many cyclic processes in nature are isochronic (Christensen, 1977; Roxburgh, 1977; Janich, 1969)

- All electrons have equal charge (Hanson, 1959)

- A transported rod is of equal length everywhere (Reichenbach, 1957; Jammer, 1959; Grünbaum, 1968; Balzer and Kamlah, 1980)

- Physical quantities are continuous (Poincarré, 1917; Schleichert, 1966)

- Physical quantities are dimensionally homogeneous (Krantz et al, 1971; Palacios, 1964; Luce, 1976; Roberts, 1980; Post, 1982)

- Coulomb's 'electrostatic density of charge' and Ohm's 'electroscopic force' refer to the same physical quantity (Heidelberger, 1980)

TABLE 1: Dichotomies relating to the semantic or epistemological status of statements about (properties of) quantities

\begin{tabular}{ll}
\hline a priori & a posteriori \\
\hline analytic & synthetic \\
normative, prescriptive & descriptive, empirical \\
rule, definition, principle & empirical law \\
mathematical, logical, formal & empirical \\
coherence; form & correspondence; content \\
convention, norm, value & fact \\
convenient or rational choice & inductive or empirical support \\
\hline
\end{tabular}

*Also: arbitrary convention, pragmatic (convenient) convention, ethical convention (or principle).
- Extensive quantities are additive (mass, velocity, utility)

- The empirical relation $>$ (for mass, length, temperature, ...) is transitive (Schleichert, 1966; Carnap, 1926; Janich et al, 1974)

- The existence of an absolute unit for velocity (a maximum velocity) (Wehl, 1949)

- The existence of an absolute zero for temperature

These are examples of statements for which it is not self-evident whether they are true (or false) by agreement $a$ priori or true (or false) by virtue of inductive support obtained a posteriori. The references in brackets refer to literature where we can find arguments supporting any of these sides. Some of the statements will be discussed in more detail below.

A related set of dichotomies is used with respect to the status of quantities and the properties of their scales. Perhaps the common denominator of this set of dichotomies is the distinction between what is basic (natural, necessary) and what are arbitrary (derived, inessential) additions. Some other related dichotomies are listed in Table 2 .

What is there to be said about these dichotomies? What is the meaning of the terms listed in the two tables in connection with measurement? Along what lines should we discuss the apparent disagreements about what is fact and what is norm, or about whether there is a need for basic, natural, absolute or fundamental quantities or units? What is the relevance of worrying about this in the first place? It would be a terrible job to analyse all details of these dichotomies. Moreover, it would be the wrong approach, I think, because the primary question to ask is: what precisely is the subject of discourse in which these dichotomies appear?

Superficial confusions can be cleared up by stating that measurement theory is a semantic theory. That is to say, measurement is considered as some sort of generalisation of giving names. By distinguishing carefully between defining

TABLE 2: Dichotomies relating to quantities and
special scale values (zero and unit)


the concept of measurement and carrying out measurements, some order can be created. However, this approach. only clears up some structural (if you want, 'logical') aspects of measurement. What is still left out is that, in the end, measurement is a social act. Measurement is carried out in a context and a measurer is just like any other decision maker: he ought to select what best serves his purposes. Apart from the formal aspects of the metric used, measurement includes operational and teleological aspects (Churchman, 1949; Adams, 1979; Gonella, 1983).

\section{The context}

Hence the crucial point is to start with the perspective that measurement is a rational activity in a context. A number of aspects of this context are of prime importance:

(a) What is the purpose of the measurement (teleological aspect)?

(b) What theoretical assumptions are given (theoretical aspect)?

(c) What are the limitations of human capabilities (operational aspect)?

(d) What are the properties of the world around us (empirical aspect?)

This whole context is, of course, dependent on the general historical and cultural context - but space does not permit a discussion of these aspects here. Aspects of (a)(d) together determine the rules of the game in the context concerned. Let us see how the perspective of measurementin-a-context elucidates some aspects of measurement.

\section{(a) The purpose of measurement}

The purpose of measurement can be quite varied, although (without going into much detail) the following distinctions can be made (Kuhn, 1961):

(i) to gather factual information to be inserted in scientific theories such as wavelengths and boiling points, but whose numerical outcome these theories to not predict;

(ii) to develop possibilities of quantitative comparison of observation by developing apparatus, reducing perturbing effects, and so on; more generally: to improve the measure of 'reasonable agreement' and to establish new measures of 'reasonable agreement' between theory and observation;

(iii) to establish the exact quantitative relation between a number of variables (of which it is already known or hypothesised that they are related); this covers a whole range from finding 'true' laws, such as Boyle's law or Hooke's law, to the establishment of empirical correlations (often using dimensional numbers) in the technical sciences and the use of multiple regression techniques to explore relations in large data sets (predominantly in the social sciences, but also in factorially designed experiments in the applied physical sciences).

(iv) only very rarely, in 'abnormal' situations, is the purpose of measurement the development or confirmation/falsification of new theories; and, if that is the case, the theoretical context of the measurement has usually been outlined long before.

As has been stressed by Kuhn (1961), established quantitative anomalies, by themselves, suggest nothing (except trouble), whereas qualitative anomalies, by their very nature, suggest at once theoretical modifications ( $a d$ hoc or otherwise). It is therefore a myth that numerical data are very likely to produce new generalisations. It is already clear from this very short analysis of the purpose of measurement that an isolated measurement result has no meaning: it is like an isolated 'fact' in history (Kushner, 1972).

\section{(b) The theoretical context of measurement}

We will find reason to refer to this dependence all through this paper: but only a few remarks at the outset. Although measurements produce 'statements of the first level', that is, 'here-thus-relations', which may suggest at once that what physics calls an object is nothing ultimately but an aggregate of characteristic numbers, this is only one side of the coin. At this lower level, the higher theoretical levels are already implied or presupposed. As Cassirer (1956) formulated it: all physical judgements, whether empirical or theoretical, mutually condition and support one another. Perhaps two categories of theoretical assump. tions made in carrying out measurement can be distinguished:

(i) Firstly, the normal situation is that a quantity is "invented' theoretically and described qualitatively (usually in terms of a set of ordering relationships) before it is or can be measured. We could here make a distinction between quantitating, which is an armchair occupation, and measuring, which is an empirical operation (Bunge, 1973). This might imply that the discovery and identification of quantities is logically independent of the discovery of a means of measurement, as is argued, for example, by Ellis (1966).

(ii) Secondly, very general assumptions are presupposed about the structure of the world. This may refer to a particular case, as when we assume that melting ice always represents the same, constant temperature. Also, they can be assumptions which bear on all measurement: for example, the assumption of a particular space-time structure.

These two categories of assumptions together structure the idealised context in which measurement is carried out.

\section{(c) Limitations of our operational capabilities}

We can roughly divide these limitations into three categories:

(i) Our capabilities of making and doing things. The logic of measurement, so to say, requires us to make exact replicas, but we can only do that approximately. If we want to assess how good we are at making replicas, we assess that by using a particular theoretical background (sometimes our ability to make something with great exactitude is better than our ability to check this high accuracy).

(ii) Our capabilities of observing differences. Using our senses we are able to judge whether one length is greater than another or that two lengths are approximately the same, but, merely going by our perceptions, the equivalence relation for length is not transitive - although the logic of measurement would like to have it that way. The psychology of sense perception may be more relevant to the foundations of measuring than is often assumed (Churchman, 1949; Horiuchi, 1983). 
(iii) Limitations of time. We cannot make instantaneous measurements or an infinite number of measurements.

(d) The empirical world and our 'objective', 'precise' and 'neutral' rendering of it

It is a myth that measurement is objective, precise, neutral. These notions only have meaning relative to a context:

(i) Precision in science and technology is always relative to a context. In spectroscopy, the accuracy of measurement is one part in one million or better; in certain parts of astronomy, a measurement which is precise as to the order of magnitude (that is to say, at most a factor of ten wrong) is extremely good. It is the theory in the discipline concerned which tells us what is 'precise' and what is 'reasonable agreement with what it should be'. It is also the theory which gives an account of the conceptual impression of a quantity. This includes such cases as the concept of temperature applied to a system consisting of a small number of molecules or the relation between different methods to measure stellar distances.

(ii) In so-called 'objective' or 'neutral' measurements, inexplicable discrepancies are often found when carried out at different places or times. This applies both when the best laboratories are involved in comparative measurements relative to a standard (mass, specific surface) or when 'constants of nature' are measured (astronomical unit, speed of light). The differences between measurements carried out at different places are considerably larger than the error bounds (obtained by conventional methods of estimating errors) would allow. Conceptual, theoretical analysis may shed light on these circumstances, as distinct from collecting yet another set of data.

(iii) The choice between a 'subjective' observer and an 'objective' instrument depends on the purpose and context of measurement (Agazzi, 1978): "what could be more vital, clearer, more obvious and more subtle than an individual's perception ... an individual is more precise, more exact, richer than the quantitative expression obtained by a measuring instrument". It is obvious that this is a different context than usually encountered in an institute of metrology. However, even when the quantity to be measured is an 'objective' one, subjective measurement may be the better option or the only possibility as, for example, in making estimates of the world energy consumption in the year 2000 using a Delphi technique.

(iv) In the physical sciences, measurements seem to be more neutral and objective than in the social sciences. In the latter case there are obvious examples that measurement results can only be objective relative to a set of normative premises. For example, the value of a price index number is only objective or neutral relative to the choice of a base period. Similarly, a poverty index is only objective relative to the normative principles that have been used to define it. Because the purpose of the physical sciences seems more generally agreed upon by natural scientists, there is perhaps less discussion about the justification of normative principles employed; but a consensus, of course, is not the same as being objective in some absolute or value-free sense.
How far have we come so far? We have disposed of the absoluteness of notions like 'exact' and 'objective' by considering them relative to a context. 'Exact' measurement takes place in an 'idealised' environment - to know what would happen under ideal circumstances requires all the science we have at our disposal. Measurement is only 'objective' in a given context and relative to a number of rules or assumptions which govern measurement in this context and which define what is considered to be 'objective' and 'precise'. These rules or assumptions are sometimes referred to as empirical axioms or laws of measurement, but also as conventions or a priori norms. An a priori norm can be, on the one hand, a 'completely arbitrary convention', or, on the other a 'value judgement'. Extreme cases are relatively easy to judge. The choice of a numerical representation is arbitrary, except for considerations of convenience. A set of axiom systems for measuring social power or poverty no doubt contains value judgements; but this is not a sharp dichotomy, and what is a rational choice, may be more or less value-loaded depending on the context.

Measurement presupposes some sort of laws or principles, that much is clear. But whether such laws are empirical, $a$ posteriori statements for which there is inductive support or whether they are, in fact, a priori normative definitions or rules, is not clear at all. The solution for this confusion or ambiguity is, I think, that what a 'rational fact' is and what a 'rational convention' depends on the theoretical context chosen. In the end all measurement rules are relative value judgements (that is, relative to the purpose of measurement).

\section{The direction of measurement}

Having eradicated most of the meaningfulness of the dichotomies commonly employed in analysing measurement by making these qualifications relative to the context, what remains? On the positive side I would like to put forward for consideration the following methodological criteria:

(a) The purpose of the science of measurement is to eliminate arbitrariness by improving upon the theoretical complexity of measurement. However, this process of elimination of as many arbitrary conventions as possible does not lead to one necessarily best solution.

(b) The methodology of the theory of measurement should be governed by an attitude of anti-fundamentalism. There are no sacred principles, necessary requirements, fundamental units, or absolute zeros.

(c) The ontology of measurement should be moderate realism, partly because (as a methodological axiom) it takes into account the context of measurement in a much more realistic way than more operationalistic or instrumentalistic stands.

I will now discuss these three criteria in more detail, showing their interrelationship and giving examples in which the relative value of the fact-norm dichotomy or the fundamental-arbitrary dichotomy is displayed.

\section{(a) Reduce arbitrariness}

A positive requirement of the methodology of measurement is: reduce arbitrariness by developing more sophisticated theoretical accounts of the magnitudes we measure. Some examples are:

(i) Reduction of the dependence of measurement (scales) on particular material bodies. This development can be 
seen in the development of the units of measurements based on anthropomorphic entities to material standards to theoretically based definitions (for example, for the unit of length). Similarly, because the choice of a material substance for a thermometer is arbitrary, one is inclined to believe that the resulting scale is of less theoretical significance than a temperature scale that would not be based on a thermometric property of a particular substance (Ellis, 1966; Mach, 1896; Ehrlich, 1982). Note that this is a methodological criterion aimed at furthering the theoretical content of the concept. It does not follow that this line is followed directly in the construction of measurement instruments where the operational criteria of calibration and accuracy predominate - however, the assessment of the validity of the instruments depends on the theoretical developments

(ii) The amount of arbitrariness is reduced if quantities turn out to have a property of having a minimum and/ or a maximum. If there is both a minimum and maximum, we may consider these values as, respectively, the zero and the unit. Examples are the measurement of angles and of velocities - and also of probability if we accept that to be a quantity existing in the real world. However, it should be stressed that a (theoretical) context is involved in establishing whether 'absolute zeros' and 'absolute units' exist - which is a point of contention subject to revision. At some point we may decide that, after all, there are velocities larger than the velocity of light or temperatures lower than the 'absolute zero', or electric charges that are not multiples of the charge of an electron.

(iii) The measurement of many physical quantities is only possible relative to a co-ordinate system, which is thereby arbitrarily introduced into the world. For example, in Newtonian mechanics the notion of absolute rest only makes sense relative to an arbitrary choice of co-ordinate system (including arbitrarily chosen units of distance). We can aim at eliminating this arbitrariness by defining the quantities of mechanics using only relations such as 'betweenness'. But, again, such a reduction of arbitrariness is never valuable in itself but only relative to a wider scope. For example, the elimination of arbitrary units and arbitrary coordinate systems in giving a synthetic treatment of mechanics and gravitational theory is proposed by Field in his book Science without Numbers (Field, 1980). Programmes which also aim at reducing arbitrariness, that ask us to conceive of all physical quantities as pure numbers, in the spirit of the old Pythagorean programme (Kroon, 1971; Whyte, 1954). Other criteria than 'reduce arbitrariness' will have to be used to decide between these two programmes.

(iv) The three examples discussed above are all very much related to the theoretical development of a concept. But, in practice we should also aim at as little arbitrariness as possible - that is to say, we should aim at giving as much theoretical justification as possible. For example, when correlating different factors in an experimental situation which is too complex to use already established theories, it is not uncommon to use any type of curve-fitting, correlation, multivariate analysis or linear regression model at hand. Assume (which is often the case) that a simple linear relationship is assumed between a concept (to be measured) and its indicators. Whatever the result is of the multiple regression technique in exploring relations in the data, if no theoretical justification can be given as to why the relationship should be linear, any conclusion that is reached is dependent for its validity upon acceptance of this arbitrary - and therefore questionable -assumption (Firestone and Chadwick, 1975).

\section{(b) Anti-fundamentalism}

Fundamentalism is construed here as any methodology which assumes that (Cassirer, 1956) (i) certain aspects of research have priority above others, (ii) this priority or precedence relieves the scientist of the obligation to investigate the 'lower' aspects. Examples of fundamentalistic attitudes are:

(1) In a scientific discipline no contradictions whatsoever are allowed.

(2) Physics is the only fundamental, exact science. A social science is possible only if founded on physics.

(3) Ethical questions cannot be evaluated rationally.

(4) A deterministic theory which explains quantum mechanics is not possible (hidden variable theories are not possible).

(5) A universal and meaningful statement about 'pure' (subjective) states of mind does not exist. And so on.

Examples of fundamentalistic statements with respect to (the possibility of) measurement are as follows:

(1) There is a fundamental distinction between fundamen tal and derived quantities in physics; the latter are only introduced for the sake of convenience (Ehrlich, 1982; Kroon, 1971).

(2) Every measurement is a derived measurement relative to the measurement of length. Therefore geometry is the most fundamental particular theory of measurement.

(3) One cannot find the measure of a sensation via its relation to the physical stimulus, because, by nature, psychophysical magnitudes cannot be reduced to space-time magnitudes (Heidelberger, 1983).

Probably the most famous and the most notorious fundamentalistic stand with respect to measurement is the following:

(4) Science is based on the measurement of fundamental quantities. A fundamental quantity is a quantity for which a physical concatenation procedure exists that leads to an additive scale and the measurement of which does not depend upon prior measurement (hence, its measurement does not depend on the measurement of other quantities. The trouble this normative demand on 'good science' has caused in the development of psychology has been well documented. Furthermore, at one time even the thermodynamic temperature scale was dismissed as incapable of measuring a 'proper' quantity (Hempel, 1952).

Enough has been said about the relevance of the context of measurement to realise that the notion of measurement being independent of anything else is a context-free abstraction with little relevance to either theory or practice. Just two examples of the influence of the context are:

(i) Whether the additive property of a physical magnitude is a matter of fact or a matter of convention depends on a priori theoretical considerations involving many different quantities. For example, the addition rule for velocities has been classified as both empirical (for 
example, based on direct measurement via the Doppler effect) and as following logically from a priori axioms of measurement. Similarly, the additivity of mass is usually considered to be an empirical law. However, if mass is defined via acceleration, the choice of an additive scale for mass is conventional (Carnap, 1926; Mach, 1869).

(ii) In the context of theoretical (as opposed to experimental) physics, it is not at all obvious that the strictly additive extensive magnitudes are basic. Actually the intensive quantities generate (by integration) extensive magnitudes (Bunge, 1973), rather than the other way round, as in $m=\int \rho(x) d^{3} x$.

So much for the interdependence of quantities. As to the additivity of fundamental quantities, there seems to be very little to support the contention that there is something essential or fundamental that would justify the demand for basing a science on additive quantities:

(i) In the early stages of the 'proper' measurement of quantities, such as mass, volume, and utility, these were considered to be additive (confirmed experimentally). However, we now know that for all three a subadditivity effect can be observed in certain circumstances. Does this make these quantities more, or less, basic? Perhaps, but only in a particular context involv. ing theories subject to revision.

(ii) Similarly velocity, which was originally additive, is not additive if interpreted relativistically. This case, together with the notorious non-additive quantity of temperature, may suggest a generalisation of the associative and commutative operation of concatenation of two objects. This is the more general property of bisymmetry (Ramsay, 1976).

$\left(a_{1} \circ a_{2}\right) \circ\left(a_{3} \circ a_{4}\right)=\left(a_{1} \circ a_{3}\right) \circ\left(a_{2} \circ a_{4}\right)$

which is a very general sort of 'averaging' property. It also covers the properties of resistance in parallelwiring and the alternative concatenation procedure for length.

(iii) Originally it was thought that any direct measurement was necessarily additive. The orthogonal (as opposed to linear) concatenation procedure for length shows that non-additive direct measurement is possible (Ellis, 1960; Van Brakel and Van der Peut, 1979). The orthogonal concatenation procedure for length also generates alternative concatenation procedures for velocity, momentum and kinetic energy (in non-relativistic physics).

\section{(c) Moderate realism}

Realism is a view about a certain class of statements for example, statements reporting the results of measurements. It is a semantic thesis: a thesis about what, in general, renders a statement in the given class true when it is true. Whether a statement is true or false depends on some reality that exists independently of our knowledge of it. This is so, independently of whether we know, or are even able to discover, its truth value. The realist position further entails (Byerly and Lazara, 1973; Dummett, 1982; Putnam, 1965):

(1) Concepts in different theories may refer to the same thing. For example, temperature is defined by the mercury thermometer, temperature as occurring in the ideal gas law, temperature as defined in thermodynamics using Carnot cycles, temperature defined as molecular energy, temperature as ascribed to a collection of (non-particulate) elementary magnets-spins, and temperature interpreted as 'felt warmness', may all refer to the same thing. It makes sense to say we are referring to the same thing, notwithstanding the fact that some of our beliefs - even beliefs included in our scientific definition of the term 'temperature' - may very likely turn out to be mistaken.

(2) Physical magnitude terms are names of magnitudes. The term 'temperature' refers to a particular physical magnitude that exists in reality and which is responsible for differences in 'felt warmness', and for certain observations Galileo made.

(3) Quantitative concepts have meaning completely apart from and independent of what measurement operations are available, or what the outcome of such measurement is.

In general, a moderate realistic position gives priority to the question of what it means for a measurement statement to be true, over and above the question of how we go about establishing its truth, or what kind of justification we can provide if we believe a measurement statement to be true. As a methodological heuristic, realism combines (i) reduction of arbitrariness and (ii) full awareness of theoretical context. This is to be counterbalanced by anti-fundamentalism. Let me give a few illustrations of this:

(i) If we assume that different concepts may refer to the same thing, this will counteract too much arbitrariness (Byerly and Lazara, 1973; Putnam, 1965). For example, it has been stated by instrumentalists and operationalists that (i) if we choose temperature to be a linear function of a mercury column this is an arbitrary choice, and (ii) the choice between $P V=R T$ and $P V=(\exp ) T$ is arbitrary. Now this may be so if we take the mercury scale or the ideal gas law in complete isolation. (In fact, it is trivial that if we remove all context everything becomes arbitrary.) But this is not so. We certainly can provide theoretical (although admittedly crude) justification for choosing a linear scale. Before we construct a scale we have knowledge about the properties of thermometric substances: bodies expand when heated; some do this more than others. Also we experience that equal increases of the length of a mercury column correspond to sensations of approximately equal temperature increases. And so on. Similarly for the ideal gas law. The variable $T$ does not occur only in connection with experiments in which $P$ and $V$ of gas samples are measured - if that were the case there would be no need for $T$ at all. We know various other (semi-) quantitative laws in which $T$ occurs. All these temperature laws are tied by reference to the same quantity. Hence the mathematical form of all these laws is tied together as well.

(ii) As has already been pointed out, we aim at a decrease of arbitrariness. For example, we consider the thermodynamic temperature scale as less arbitrary than a scale based on a thermometric substance, and we do this for good theoretical reasons. However, I think it is a sign of fundamentalism if we call the thermodynamic scale an absolute scale. As Berka (1983) writes: "An arbitrary zero as well as the absolute zero are theoretical constructs that are neither absolutely arbitrary nor absolutely natural". In particular in connection with the 'absolute zero' for temperature, very strong opinions have been expressed in the literature, for example:

(a) The ideal gas scale cannot help having an absolute zero since it will not make sense to talk of cooling 
a given mass of gas down by more degrees of temperature below $0^{\circ} \mathrm{C}$ than will reduce this pressure to zero (Ellis, 1966; Byerly and Lazara, 1973; Toulmin, 1960). But: what good reason is there to call this zero absolute and to capitalise this phrase? Why would the ideal gas law be valid at 'absolute zero' (after all, no gases exist at $-273.15^{\circ} \mathrm{C}$ )?

(2) The whole structure of the science of thermodynamics would collapse without the existence of this (fixed, but unattainable) lower limit of temperature (Sommerfeld, 1956). But the science of thermodynamics did not collapse, nor did the concept of temperature fragment when the notions of negative, infinite, and hotter than infinite temperatures gained legitimacy in quantum statistical mechanics (Ehrlich, 1982).

Perhaps you may object that it is impossible to talk about hotter than infinite temperatures. I would argue against that as it being a fundamentalistic position forbidding certain lines of research. Whether it makes sense to refer to hotter than infinite temperatures or temperatures below absolute zero is a sophisticated subject of discussion in the foundation of quantum-statistical mechanics. Perhaps this approach leads to a 'less arbitrary' account of the concept of temperature. Whatever the outcome may be, it does not mean that the reference to the term 'temperature' will depend on it.

\section{Conclusion}

First, measurement is measurement-in-a-context. This context consists of our purpose; what we know; what we can do, the empirical world.

Secondly, it follows that which rules of measurements are facts or norms depends on the whole theoretical background we have rationally decided to adhere to.

Thirdly, sensible guidelines for a theory of measurement are: reduce arbitrariness; no fundamentalism; do not be afraid of realism.

\section{References}

Adams, E. W. 1979. Measurement theory, in current research in philosophy of science, P. D. Asquith and H. E. Kyburg, eds, 207-227.

Agazzi, E. 1978. 'Is scientific objectivity possible without measurements?' Diogenes, 104, 93-111.

Balzer, W. and Kamlah, A. 1980. 'Geometry by ropes and rods', Erkenntniss, 15, 245-267.

Berka, K. 1983. Measurement: Its concepts, theories and problems, Reidel, Dordrecht.

Bunge, M. 1973. 'On confusion measure with measurement in the methodology of behavioural science', in The methodological unity of science, M. Bunge, ed, Reidel, Dordrecht, 105-122.

Byerly, H. C. and Lazsra, V. A. 1973. 'Realist foundations of measurement', Philosophy of Science, 40, 10-28.

Carnap, R. 1926. Physikalische Begriffsbildung, Braun, Karlsruhe.

Cassirer, E. 1956. Determinism and indeterminism in modern physics, Yale University Press, New Haven.

Christensen, F. 1977. 'How to establish non-conventional isochrony, Brit J Phil Sci, 28, 49-54.

Churchman, C. W. 1949. 'A materialist theory of measurement', in Philosophy for the future, R. W. Sellars et al, eds, MacMillan, New York.

Dummett, M. 1982. 'Realism', Synthese, 52, 55-112.

Ehrlich, P. 1982. 'Negative, infinite and hotter than infinite temperatures', Synthese, 50, 233-277.
Ellis, B. 1960. 'Some fundamental problems of direct measurement', Australian Journal of Philosophy, 37-47.

Ellis, B. 1966. Basic concepts of measurement, Cambridge University Press.

Field, H. H. 1980. Science without numbers, Basil Blackwell, Oxford.

Firestone, J. M. and Chadwick, R. W. 1975. 'A new procedure for constructing measurement models of ratio scale concepts', Int J General Systems, 2, 35-..53.

Gillies, D. A. 1973. An objective theory of probability, Methuen, London.

Gonella, L. 1983. 'Operative approach to the theory of measurement', in Proceedings of the Workshop on Fundamental Logical Concepts of Measurement, 5-6 September 1983, Turin, IMEKO and the Instituto di Metrologia ' $G$. Colonnettii',

Grübaum, A. 1968. Geometry and chronometry in philosophical perspective, University Press of Minnesota.

Grunstra, B. R. 1969. 'On distinguishing types of measurement', Boston studies in the philosophy of science, $\mathbf{5}$, R. S. Cohen and W. Wartofsky, eds.

Hanson, N. R. 1959. Patterns of discovery, Cambridge University Press.

Heidelberger, M. 1980. 'Towards a logical reconstruction of revolutionary change: the case of Ohm as an example', Stud Hist Phil Sci, 11, 103-121.

Heidelberger, M. 1983. 'Philosophische Konzeptionen der Messung im 19 Jahrhundert', paper presented in section 13 of the 7th Int Congress of Logic, Methodology and Philosophy of Science, Abstracts, 87-88.

Hempel, C. G. 1952. Fundamentals of concept formation in empirical sciences, University of Chicago Press.

Horiuchi, T. 1983. 'Errors and uncertainty in the framework of representational measurement theory and some scopic classes of error sources', Proc Workshop Fundamental Logical Concepts of Measurement, L. Gonella, ed, Instituto di Metrologia ' $\mathrm{G}$. Colonnetti', Turin.

Jammer, M. 1959. Concepts of Space, Harvard University Press, Cambridge.

Janich, P. 1969. Die Protophysik der Zeit, Bibliographisches Institut, Mannheim

Janich, P., Kabartel, F. and Mittelstrass, J. 1974. Wissenschaftstheorie als Wissenschaftskritik, Aspekte Verlag, Frankfurt-am-Main.

Krantz, D. H., Luce, R. D., Suppes, P. and Tversky, A. 1971. Foundations of measurement, vol I, Academic Press, New York/London.

Kroon, R. P. 1971. Dimensions, Joumal of the Franklin Institute, 292, 45-55.

Kuhn, T. S. 1961. 'The function of measurement in modern physical science', ISIS, 52, 161-190.

Kushner, L. M. 1972. 'The role and value of measurement', Proc of the 1972 Joint Measurement Conference, Boulder, California.

Luce, R. D. 1978. 'Dimensionally invariant numerical laws correspond to meaningful qualitative relations', Phil of Sci, 45, 1-16.

Mach, E. 1868. 'Uber die Definition de Masse', Repertorium für Physikalische Technik, 4, 355-359.

Mach, E. 1896. Die Principien der Wärmelehre, Leipzig, 39-57; also in English in Ellis (1968), 183-196.

Nagel, E, 1968. The structure of science, Routledge \& Kegan Paul, London.

Osborne, D. K. 1976. 'Unified theory of derived measurement', Synthese, 33, 455-481.

Palacios, J. 1964. Dimensional analysis, Macmillan, London; 1 st edn 1956, Espana-Calpe, Madrid. 
Poincaré, H. 1917. La science et l'hypothese, Flammarion, Paris.

Post, E. J. 1982. 'Physical dimensions and covariance', Found Physics, 12, 169-195.

Putnam, H. 1965. "How not to talk about meaning: Comments on J. J. C. Smart, in Boston studies in the philosophy of science, M. W. Wartofsky and R. S. Cohen, eds, Vol 2, Humanities Press, New York; also published in Putnam, H. 1975. Philosophical papers, Vol 2, $117-$ 131.

Putnam, H. 1973. Explanation and reference, in conceptual change, G. Pearce and P. Maynard, eds, Reidel, Dordrecht, 199-221; also published in Putnam, H. 1975. Philosophical papers, Vol 2, 196-217.

Ramsay, J. O. 1976. 'Algebraic representation in the physical and behavioural sciences, Synthese, 33, 419-453.

Reichenbach, H. 1957. The philosophy of space and time, Dover, New York.
Roberts, F. R. 1980. 'On Luce's theory of meaningfulness', Philosophy of Science, 47, 424-433.

Roxburgh, I. W. 1977. 'The conventionality of uniform time', Brit J Phil Sci, 28, 172-177.

Schleichert, H. 1966. Elements der physikalischen Semantik, Oldenbourg, Vienna.

Sommerfeld, A. 1956. Thermodynamics and statistical methods, Harper Torchbooks, New York.

Toulmin, S, 1960. Philosophy of science, Harper Torchbooks, New York.

Van Brakel, J. and Van der Peut, A. A. 1979. 'Alternative physical concatenation procedures in length measurements', Abstracts 6th Int Congress of Logic, Methodology and Philosophy of Science, sections 8 and 9, 36-40.

Weyl, H. 1949. Philosophy of mathematics and natural science, University Press, Princeton.

Whyte, L. L. 1954. 'A dimensionless physics?' Brit J Phil Sci, 5, 1-17.

\title{
6th Hungarian Conference on Bio-medical Engineering and 2nd IMEKO Conference on Measurement in Clinical Medicine
}

\author{
Balatonfüred, Hungary, 18-21 September 1984
}

The scientific sessions will demonstrate the latest results in biomedical engineering and will give a survey of the main trends of the field. The conference will be accompanied by a commercial/ scientific exhibition.

\section{Scope}

- The acquisition of signals corresponding to physiological and clinical variables

- Processing of physiological and medical signals and images

- Model-based inferential measurement, for physiology and medicine

- Integration of measurement and control in model-based diagnosis, clinical decision making, patient management and automatic control of drug administration

- New methods and instruments in clinical engineering and therapy

- New developments in health care and its organisation

\section{Main sections}

1. Sensors, transducers, measurement of physiological signals

2. Signal processing

3. Radiology, nuclear medicine

4. Processing of medical images

5. Modelling, simulation, decision making, control applications

6. Clinical and hospital engineering

7. Perinatology

8. Health care systems

9. Education and training

Working language: English (with simultaneous translation into Hungarian).

Correspondence related to the Conference should be addressed to the Scientific Society of Measurement and Automation (MATE), 1055 Budapest, Kossuth Lajos ter 6-8, Hungary, or to the IMEKO Secretariat, 1371 Budapest, POB 457, Hungary (Phone: (36-1) 324 116; Telex: 225792 mtesz h). 